\title{
Prima e dopo Lamarck. Il miglioramento della specie umana tra ereditarietà e degenerazione
}

Germana Pareti

\section{(2) OpenEdition}

\section{Journals}

\section{Edizione digitale}

URL: http://journals.openedition.org/studifrancesi/4263

DOI: 10.4000/studifrancesi.4263

ISSN: 2421-5856

Editore

Rosenberg \& Sellier

\section{Edizione cartacea}

Data di pubblicazione: 1 settembre 2016

Paginazione: 216-232

ISSN: 0039-2944

\section{Notizia bibliografica digitale}

Germana Pareti, « Prima e dopo Lamarck. II miglioramento della specie umana tra ereditarietà e

degenerazione », Studi Francesi [Online], 179 (LX | II) | 2016, online dal 01 septembre 2017, consultato il 18 septembre 2020. URL : http://journals.openedition.org/studifrancesi/4263 ; DOI : https://doi.org/ $10.4000 /$ studifrancesi.4263

\section{(c) (i) (9)}

Studi Francesi è distribuita con Licenza Creative Commons Attribuzione - Non commerciale - Non opere derivate 4.0 Internazionale. 


\title{
Prima e dopo Lamarck. Il miglioramento della specie umana tra ereditarietà e degenerazione
}

\begin{abstract}
This paper aims to illustrate the relationship between heredity and degeneration in the nineteenth-century French scientific culture. From the work of P. Lucas on inheritance in nervous diseases, a large group of psychiatrists, followers of Pinel and Esquirol, began to investigate inheritance in madness. For Morel, however, the transmission of madness did not consist purely of a mechanism based on inheritance. On the contrary, nervous disease is a real degenerative form, which resolved in the extinction of the family line. Among the causes of the dégénérescence alienists indicated especially social conditions: the recent industrialization and urbanization yielded many social plagues. Typically, those who showed the stigmata of hereditary defects included among its own ancestors subjects addicted to vice. Consequently doctors and psychiatrists proposed programs of hygiene and public health. At the same time, the mechanisms of variation and inheritance were investigated by breeders and farmers. Darwin recognized their results, but only the revolution in genetics could put an end to the mystery of heredity.
\end{abstract}

\section{Ereditarietà e degenerazione}

«L'hystérie n'est-elle pas un mode de dégénérescence?»

«L'hystérie est fréquemment une maladie d'hérédité, et rien n'est commun comme de voir l'hystérie se develop sur un fond de dégénérescence...»'

La triade "isteria-follia-degenerazione mentale" era un tema caldo per gli alienisti e i neurologi francesi riunitisi a congresso (il quinto) nel 1894 a Clermont-Ferrand. Sul carattere ereditario dell'isteria insisteva il presidente del convegno, Gilbert Ballet, affiancato dal neurologo Alix Joffroy, allievo di Charcot e titolare della cattedra di "Clinique des maladies mentales et de l'encéphale" al Sainte-Anne di Parigi. Emmanuel Régis, invece, ne proponeva una connotazione morale: «la perversité morale des hystériques existe», domandandosi se «y a-t-il coexistence de l'hystérie et de la dégénérescence, ou bien l'hystérie est-elle une forme de dégénérescence?» ${ }^{2}$. Non tutti gli esperti però erano convinti del binomio “isteria-degenerazione”. Per Jules Falret, figlio dello psichiatra Jean-Pierre e grande estimatore di Charcot, «le mot dégénérescence est un terme trop vague qui ne donne pas l'explication des faits». Non così per Ballet, che nello speciale Rapport de l'bystérie et de la folie aveva svelato l'inquietante tendenza al suicidio negli isterici, propensione nella quale si potevano cogliere le stigmate dell'ereditarietà.

(1) Chronique. Le Congrès des médecins aliénistes et neurologistes de France et des pays de langue française, à Clermont Ferrand, «Annales médico-psychologiques» LII (t. XX), 1894, pp. 225 e 223.

(2) Ivi, p. 224 
Che suicidio e dégénérescence fossero segnali di sofferenze nervose era una tesi formulata, trent'anni addietro, da parte di un neurologo tedesco non particolarmente affezionato all'interpretazione degenerativa. In una raccolta di casi clinici comprovanti la "predisposizione ereditaria" alla follia, Wilhelm Griesinger, all'epoca primario di Clinica psichiatrica alla Charité di Berlino, citava un fatto storicamente significativo $^{3}$. Già menzionato da Jean-Baptiste Lautard nel famoso saggio storico-statistico La Maison des Fous de Marseille (1840), questo case study portava sostegno alla tesi che «l'hérédité était la cause la plus ordinaire de la folie», «une cruelle manière d'être [...] se trasmettant d'une génération à l'autre» ${ }^{4}$. La fatale concatenazione si ravvisava nella tragica discendenza di due coniugi morti entrambi suicidi, la cui progenie era segnata dal marchio della pazzia: una figlia prostituta, suicida col veleno; un maschio, strangolatosi dopo un'accusa di omicidio; un'altra figlia minore, anch'essa suicida incinta di sei mesi, del cui erede si erano perse le tracce in Egitto, dopo un lungo periodo trascorso in prigione.

A partire dal 1857, anno di pubblicazione del Traité des dégénérescences physiques, intellectuelles et morales de l'espèce bumaine et des causes qui produisent ces

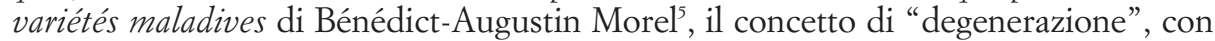
tutte le sue varianti lessicali, aveva rappresentato uno strumento diagnostico potente, che sembrava mettere d'accordo alienisti di diversa provenienza formatisi nelle scuole di Philippe Pinel e Jean-Étienne Esquirol. L'applicazione di questo modello interpretativo poté contare anche sulla statistica recentemente introdotta. La narrazione di casi singoli e gli elenchi di dati biografici e di alberi genealogici lasciavano il posto alla rielaborazione statistica dei dati empirici che i medici ricavavano dal vasto repertorio dei ricoverati nelle case di cura ${ }^{6}$. Le tavole statistiche consentivano di "monitorare" l'andamento e la progressione delle malattie mentali in concomitanza con determinati fenomeni sociali. Ma già tredici anni prima della pubblicazione del trattato di Morel, nelle Annales médico-psychologiques del 1844, un allievo di Esquirol, Jules Baillarger, aveva cercato di determinare con strumenti quantitativi la proporzione della follia ereditaria rispetto ai casi di quella non ereditaria.

In queste ricerche furono introdotti i quesiti canonici sull'ereditarietà nelle malattie mentali. La follia materna è trasmessa più frequentemente per via materna o paterna? Si trasmette in linea diretta "per genere", cioè preferenzialmente dalla madre alle figlie e viceversa, dai padri ai maschi? ${ }^{7}$ All'epoca medico interno alla Salpêtrière, Baillarger poteva contare su 600 osservazioni, da lui riassunte in 38 tavole statistiche, che "fotografavano" la situazione della trasmissione in linea diretta e collaterale. In ben 453 di quei casi era evidente la "linea" materna: «la folie de la mère est donc plus fréquemment héréditaire que celle du père dans la proportion d'un tiers». Essa "colpisce" un maggior numero di figli e, mentre i maschi ereditano la follia suppergiù tanto dalla madre quanto dal padre, le femmine invece ereditano (almeno) due volte più frequentemente dalla madre che dal padre.

(3) W. GRIESINGER, Die Pathologie und Therapie der psychischen Krankheiten (Stuttgart, Krabbe, 1845). Dall'edizione tedesca $\left(1861^{2}\right)$ furono pubblicate la traduzione francese (Traité des maladies mentales. Pathologie et thérapeutique, Paris, Delahaye, 1865) e quella inglese, dalla quale d'ora innanzi si citerà (Mental Pathology and Therapeutics, London, The New Sydenham Society, 1867, rist. anastatica 2008).

(4) J.-B. Lautard, La Maison des Fous de Marseille. Essai historique et statistique, Marseille, D'Achard, 1840, p. 120.
(5) Paris, Baillière, 1857.

(6) Cfr. C. López-Beltrán, Storytelling, Statistics and Hereditary Thought: the Narrative Support of Early Statistics, «Studies in the History and Philosophy of Biological and Biomedical Sciences» XXXVII, 2006, pp. 41-58.

(7) J. BAILlARGER, Recherches Statistiques sur l'Hérédité de la Folie, «Annales médicopsychologiques», III, 1844, pp. 328-339.

(8) Ivi, p. 331. 
Dunque l'ereditarietà nella follia sembrava confutare «l'opinion populaire du croisement des sexes dans la génération»", tesi a favore della quale si erano espressi gli autorevoli Karl Burdach e Albrecht von Haller. Burdach citava Fabrizio di Acquapendente, che aveva distinto gotta e cachessia (ereditabili dal padre) da spasmi, melancolia, vivacità e facoltà intellettuali, trasmissibili per via materna. Altri casi comprendevano anomalie di organizzazione cerebrale trasmesse dalle madri ai maschi $\mathrm{o}$, viceversa, dai padri alle figlie, oppure l'eredità delle doti e delle qualità superiori, che si pensava fossero ereditate dai grandi uomini in virtù del cosiddetto «esprit maternel» $\gg^{10}$. Ma sulla possibilità di questi incroci, soprattutto nella follia, Baillarger era cauto, giacché la maggior frequenza dell'eredità da parte materna era «désormais un argument de plus en faveur de tous ces faits» ${ }^{11}$.

Sebbene la storia delle malattie ereditarie fosse «très peu avancée», Baillarger era convinto che le nuove conoscenze sul sistema nervoso avrebbero gettato luce anche sull'ereditarietà, poiché si trattava del «résultat de la transmission d'une certaine organisation cérébrale» ${ }^{12}$. Le future ricerche in fisiologia patologica non si sarebbero limitate, però, a trattare i soli aspetti patologici dell'ereditarietà ${ }^{13}$. Con un atteggiamento che si sarebbe diffuso tra i neurofisiologi del secolo seguente, Baillarger riteneva che i casi clinici concorressero a spiegare i meccanismi dei processi nervosi normali, per esempio le funzioni cognitive e la trasmissione delle facoltà morali e intellettuali. Tuttavia le malattie nervose godevano di un privilegio. Le patologie ereditarie "classiche" (tisi, scrofula, gotta ecc.) non davano informazioni sulle cause di morte dei progenitori, impedendo di stabilire qualsivoglia rapporto causale diretto con (eventuali) forme morbose degli avi. Per il fatto di non essere «disséminés ça et là», bensì riuniti in apposite case di cura, gli alienati costituivano invece una fonte inesauribile di osservazione e controllo. Gli oltre duemila ricoverati negli asili parigini mettevano a disposizione un numero crescente di informazioni dettagliate sui loro genitori e sulle cause della loro degenerazione e morte. Dunque, nessun tema più dello studio della follia poteva aiutare a delineare il quadro e la progressione della storia generale delle malattie ereditarie.

Nel triennio successivo (1847-1850) apparvero i due volumi del Traité Philosophique et Physiologique de l'Hérédité Naturelle dans les États de Santé et de Maladie du Système Nerveux a firma di Prosper Lucas ${ }^{14}$. Lucas si era addottorato in medicina a Parigi nel 1833 ed era entrato come psichiatra prima a Bicêtre, quindi al Sainte-Anne. Le notizie biografiche su questo medico francese nato nel 1808 e molto attivo negli eventi del 1848, sono piuttosto scarne. Di lui si sa che scrisse poco, fatta eccezione per i due ponderosi tomi (rispettivamente di 623 e 929 pagine) del trattato sull'eredità, che intendeva forse completare con un terzo volume ${ }^{15}$. Il Traité in effetti è una sorta di zibaldone di dottrine talora strampalate, che spaziano dalla filosofia all'embriolo-

(9) Ivi, p. 336.

(10) Ivi, p. 135.

(11) Ibid.

(12) Ivi, p. 334. A Baillarger si deve la prima formulazione dell'idea di un'organizzazione stratificata laminare della corteccia cerebrale.

(13) J. Baillarger, Recherches su l'anatomie, la physiologie et la pathologie du système nerveux, Paris, Masson, 1847, Introduction, p. XXIII.

(14) In realtà il titolo è ben più chilometrico e recita: Traité Philosophique et Physiologique de l'Hérédité Naturelle dans les États de Santé ed de Maladie du Système Nerveux, avec l'Application Méthodique des Lois de la Procréation au Traitement
Général des Affections dont elle est le Principe. Ouvrage où la Question est considerée dans ses Rapports avec les Lois Primordiales, les Théories de la Génération, les Causes Déterminantes de la Sexualité, les Modifications Acquises de la Nature originelle des Etres, et les Diverses Formes de Névropathie et d'Aliénation Mentale (2 voll., Paris, Baillière, 1847-1850).

(15) L'opera di questo semisconosciuto medico francese è recente oggetto di studi nell'Università di Città del Messico, cfr. R. Noguera-Solano e R. RuIZ-GuTIÉRREZ, Darwin and Inberitance: The Influence of Prosper Lucas, «Journal of the History of Biology», 2009, online-first. 
gia, dalla zoologia alla Kabala, dalla morale alla fisiologia. Un distillato di oltre 1500 pagine delle idee sviluppate dall'antichità fino al primo Ottocento sul concetto di ereditarietà e sulle sue applicazioni non soltanto nelle scienze della vita, ma anche in altri svariati campi del sapere, dalla politica alle scienze sociali. Lucas mirava a conciliare i dogmi della fissità e dell'invariabilità delle specie con i fenomeni di variazione e sviluppo individuale in ogni singola specie ${ }^{16}$. A tal fine andavano chiarite (e soprattutto distinte) le leggi della procreazione e dell'ereditarieta ${ }^{17}$, e individuate le anomalie a carico soprattutto del sistema nervoso. In particolare, la legge dell'innéité giustificava la variabilità individuale all'interno della specie («l'unique principe de cette force qui tend à la diversité dans la génération ${ }^{18}$ ) mentre quella dell'ereditarietà «répresente ce qu'il y a de répétition et de mémoire de la vie» ${ }^{19}$. Ispirandosi alla fisiologia di Johannes Müller e al vitalismo dell'epoca, Lucas era convinto che con queste sole due leggi fosse possibile spiegare tutte le differenze individuali, interne ed esterne, quelle della costituzione individuale e quelle causate dalle influenze ambientali.

A cavallo tra i due secoli, la questione dell'ereditarietà delle modificazioni acquisite restava «une des plus importantes et vastes de la physiologie générale» ${ }^{20}$, secondo il parere di Georges Cuvier riportato da Marie-Jean-Pierre Flourens. Anche per Lamarck:

Tout ce que la nature a fait acquérir ou perdre aux individus, par l'influence des circonstances où leur race se trouve depuis longtemps exposée, [...] elle le conserve par la génération aux nouveaux individus qui en proviennent, pourvu que les changements acquis soient communs aux deux sexes ou à ceux qui ont produit ces nouveaux individus ${ }^{21}$.

Ma non tutti gli studiosi erano d'accordo sull'ereditarietà delle modificazioni acquisite. Pur citando il giudizio di James C. Prichard, secondo il quale, per quanto l'ambiente fosse in grado di modificare la costituzione individuale, i cambiamenti non potevano considerarsi trasmissibili («se bornent à l'individu et n'ont point d'influence sur sa postérité» ${ }^{22}$ ) Lucas non ne era convinto. Oltre che delle idee del trio CuvierBuffon-St.-Hilaire, egli faceva tesoro anche delle risultanze degli esperimenti condotti dagli ibridatori e allevatori inglesi sulle razze pregiate di cani e cavalli. Tra questi Charles Girou de Buzareingue ${ }^{23}$. Nella trasmissione agiva la «force séminale de la propagation» dei caratteri acquisiti, distinti secondo le tre età della vita, relativamente a forme, colore, proporzioni, funzioni dipendenti dalle abitudini ecc., comprese le modificazioni di natura morale e quelle risultanti dall'educazione e dall'istruzione.

Rivelatore è il raffronto tra i manoscritti di Darwin precedenti la pubblicazione dell'Origin of the Species e l'opera di Lucas. Pare che Darwin avesse letto Lucas fin dal settembre $1856^{24}$. Nel novembre sarebbe giunto a formulare "il" problema fondamentale che ruotava intorno alle nozioni di variabilità ed ereditarietà, e cioè se le nuove strutture fossero trasmissibili. Sempre dopo la lettura di Lucas, Darwin avrebbe adottato nel proprio lessico il termine inheritance, codificandolo tuttavia soltanto una decina di anni più tardi, nel 1868 in The Variation of Animals and Plants under Domestication.

(16) LuCAs, Traité cit., I vol. p. 171.

(17) Ivi, vol. I, p. XVI.

(18) Ivi, vol. II, p. 442.

(19) Ivi, vol. I, p. 96.

(20) Ivi, vol. II, p. 456.
(21) La presente citazione di Lamarck (Philosophie zoologique, t. I, cap. VIII, p. 235) è in LuCAS, Traité cit., vol. II, p. 458.

(22) Ivi, II vol. p. 457.

(23) LuCAS, Traité cit., vol. I, p. 203 e ss.

(24) Cfr. Correspondence, vol. IV, appendix IV. 


\section{L'hérédité e le sue forme}

Fin dal primo Settecento, e non solo in ambiente medico, il concetto di "trasmissione ereditaria” era discusso nel contesto delle teorie razziali. John Ray (1691), Johann Friedrich Blumenbach e, successivamente, antropologi e naturalisti soprattutto inglesi si sforzavano di conciliare l'idea della creazione divina con quella della diversità delle specie e delle variazioni più o meno accidentali ${ }^{25}$. Le stesse variazioni ottenute attraverso l'addomesticamento, l'allevamento e la coltivazione al fine di preservare le caratteristiche desiderate in una specie possono essere interpretate come il prodotto della tensione tra "natura e cultura", che caratterizzava lo sperimentalismo dell'epoca. Ovviamente questa discussione si rifletteva sulla specie umana, nella quale, a differenza di quanto accadeva per talune deficienze organiche o di linguaggio, sussistevano certe peculiarità dei caratteri acquisiti che non venivano trasmesse alla discendenza. Per Blumenbach, le diverse varietà dell'uomo risalivano a un'unica e stessa specie ${ }^{26}$, e pertanto la "degenerazione" (termine al quale non attribuiva un significato negativo) si limitava a una variazione da "specie primitive". Nell'area culturale anglosassone, i cosiddetti "pre-darwiniani" (tra cui il già menzionato Prichard, William Lawrence e William Charles Wells) si mostravano scettici circa l'ereditarietà dei caratteri acquisiti e le loro idee si diffusero tra i medici e gli antropologi.

Non è un caso che nel 1835 Prichard avesse descritto le malattie mentali in un trattato dedicato a Esquirol. Citando i pareri di svariati psichiatri francesi, riconosceva che in molte forme di "pazzia morale" esisteva in famiglia «an hereditary tendency to madness $\gg^{27}$. Con Prichard incomincia a prendere forma la figura del medico-antropologo-scienziato dedito allo studio della trama tortuosa della "mente", o meglio del cervello, sede di affezioni che manifestano la complessità dell'intreccio tra ereditarietà, variazioni e malattie nervose. In questo contesto, prevedibilmente, le malattie mentali rappresentavano le forme morbose con i segni "canonici" più eclatanti del decadimento fisico e morale ${ }^{28}$.

I naturalisti e i medici settecenteschi avevano osservato il fenomeno della ripetizione di una variazione nella discendenza. Sulle variazioni incidevano sia la casualità sia le influenze causali esterne. Dall'insieme di questi fattori era possibile calcolare la probabilità dell'apparizione della variazione spontanea nella prole. Paradigmatici furono il caso della famiglia Ruhe di Berlino "in osservazione" per il ripetersi del polidattilismo nella discendenza a partire dall'antenata che per prima aveva presentato questo tratto straordinario ${ }^{29}$, e quello dei Lambert, la famiglia degli uomini-porcospi-

(25) Cfr. M. Banton, Racial Theories, Cambridge, Cambridge University Press, 1987 e i saggi contenuti in Race, Science and Medicine, 1700-1960, a cura di W. ERnST e B. HARRIS, London, Routledge, 1999 nonché in Race and Racism in Modern Philosophy, a cura di A. VALLs, Ithaca (N.Y.), Cornell University Press, 2005. Sui "pre-darwiniani", cfr. K.D. WELLS, Sir William Lawrence (1783-1867). A Study on pre-Darwianian Ideas on Heredity and Variation, «Journal of the History of Biology» IV, 1971, pp. 319-361.

(26) J.F. BlumenBach, The Anthropological Treatises of Johann Friedrich Blumenbach: De Generis Humani Varietate Nativa (1795), trad. ingl. a cura di T. Bendyshe per l'«Anthropological Society of London», London, Longman, Green, Longman, Roberts \& Green, p. 275.

(27) J.C. PRICHARD, Treatise on insanity and other disorders affecting the mind, London, Sherwood,
Gilbert and Piper, 1835, p. 12, nonché pp. 23 e 36 e cap. IV, sezione II: "Predisposing Causes of Insanity", passim.

(28) Questo giudizio era sostenuto già nella tesi di dottorato (1992) di C. López-Beltrán, Human Heredity 1750-1780: The Construction of a Domain, e ribadito in ID., Forging Heredity: from Metaphor to Cause, a Reification Story, «Studies in History and Philosophy of Science» 25 (2), 1994, pp. 211235; cfr. inoltre S.M. QuINLAN, Inheriting Vice, Acquiring Virtue: Hereditary Disease and Moral Hygiene in the Medicine of the French Enlightenment, «Bulletin of the History of Medicine» LXXX (4), 2006, pp. 649-675.

(29) Sulla "fortuna" di questo caso in Pierre L. Moreau de Maupertuis (Lettre XIV, 1752, p. 308), cfr. I. SAndler, Pierre Louis Moreau de Maupertuis - A Precursor of Mendel?, «Journal for History of Biology» XVI, 1983, pp. 101-136. 
no affetti da una gravissima forma di ipercheratosi. Un membro di questa famiglia fu esibito persino alla Royal Society ${ }^{30}$. Da un lato, lo studio di questi fenomeni serviva a stabilire la probabilità della convergenza di aberrazioni casuali nelle fasi di sviluppo embriologico degli appartenenti a una stessa famiglia. Da un altro lato, recava nuovi elementi al dibattito tra preformisti ed epigenisti: per questi ultimi, al momento della formazione, il principio morboso si mescolava con il germe seminale e, nel corso dello sviluppo, poteva dar origine a una malattia, a un cattivo temperamento o a una semplice disposizione.

Frattanto varie istituzioni e accademie francesi (a partire da quella di Digione nel $1748^{31}$ e la Société Royale de Médecine nel 1787) invitarono gli esperti a intervenire sul tema, trovando risposte a vari questionari. Esistevano malattie «vraiement héréditaires»? Si invitava pertanto a redigerne un elenco, suggerendo strategie per impedirne lo sviluppo e favorirne la guarigione. Nel $1790 \mathrm{fu}$ bandito un nuovo concorso dalla Société Royale de Médecine ${ }^{32}$, dopo che erano stati giudicati insoddisfacenti i precedenti lavori pervenuti ${ }^{33}$. Dalle risposte emergeva che le malattie ereditarie non solo esistevano, ma che costituivano un allarmante segno di degenerazione e declino della nazione, sintomo che raggiungeva la massima espressione nelle malattie mentali.

La voce "Héréditaire" apparsa nel Dictionnaire des Sciences Médicales a firma di Antoine Petit condensava un insieme di riflessioni già esposte in saggio apparso nello stesso anno ${ }^{34}$. L'aggettivo "héréditaire" designava «une circonstance particulière à quelques maladies» che consiste «dans une certaine disposition organique, que les parents, qui ont atteints ces maladies, transmettent à leur enfants par voie de génération ${ }^{35}$. Secondo Petit, le malattie ereditarie si manifesterebbero nella progenie generalmente alla stessa età e nel corso delle medesime circostanze nelle quali si erano espresse nei genitori e, se pure "saltavano" una generazione, si ripresentavano però in quella successiva, talora accentuate o ritardate da determinati fattori. Tra le possibili cause, Petit scartava l'ipotesi di un virus avanzata dal presidente della Société Royale de Médecine, Antoine Portal. Petit confutava anche i pareri di Buffon e di Charles Bonnet, per i quali, se non sono sane le forme interiori, anche le molecole organiche sono attaccate dal vizio e affettano gli umori e, di conseguenza, il liquido seminale. All'economia del corpo organizzato meglio si adattava l'ipotesi della predisposizione. Tra le cause predisponenti, oltre a quelle fisiche, Petit annoverava quelle morali, che agiscono in maniera da rendere la disposizione «partie inhérente de l'individu» ${ }^{36}$, addirittura «une condition harmonique» della sua esistenza.

A Parigi, negli interventi di Portal, Petit e François-Emanuel Fodéré sull'ereditarietà fisica e morale si fronteggiavano le opinioni dei rivali savants che Buffon aveva chiamato al Jardin du Roi. Costoro non perdevano occasione di fomentare polemiche. Ma al di là delle invidie professionali, questo contrasto era una coda dello scontro tra due opposte concezioni del corpo, solidistica e umoralistica, risalenti alla

(30) E.A. COCKAYNE, Inherited Abnormalities of the Skin and Its Appendages, London, Oxford University Press, 1933.

(31) Cfr. C. LóPez-Beltrán, "Les maladies béréditaires": $18^{\text {th }}$ century disputes in France, «Revue d'histoire des sciences» XLVIII, 1995, pp. 307-350, spec. p. 311 ss. e ID., The Medical Origins on Heredity in Heredity Produced (a cura di S. Müller-Wille e H.-J. Rheinberger), Cambridge (Mass.), The MIT Press, 2007, pp. 105-132.

(32) Cfr. Prix distribués et proposés dans la séance publique de la Société royale de médecine, tenue au
Louvre le 31 août 1790. Prix distribués. 1- Maladies béréditaires.

(33) Sulla politica condotta dalla Société Royale nell'ambito del programma sanitario della nazione francese, cfr. QUINLAN, Inheriting Vice cit. p. 670.

(34) A. Petit, Essai sur les Maladies Héréditaires, Paris, Gabon, 1817.

(35) «Héréditaire», in Dictionnaire des Sciences Médicales, tome 21, Paris, Panckoucke, 1817, pp. 58-86, p. 58.

(36) Ivi, p. 75. 
medicina del passato. Alla predisposizione ereditaria, in termini di diatesi, si contrapponeva l'azione di "cause esterne" e contagiose di malattia, anche se queste ultime non giustificavano i disturbi cronici che potevano presentarsi a carico dei membri di una stessa famiglia.

I medici della generazione successiva aspirarono ad assumere un ruolo sempre più importante nella gestione dei programmi di igiene pubblica. Prese piede il "medico morale", studioso attento ai mali non soltanto individuali ma soprattutto sociali, e pertanto sensibile alla rigenerazione della società. A tal fine si richiedevano programmi specifici di salute fisica e morale $e^{37}$. La medicina mentale era "etica", poiché doveva combattere la follia, malattia tipicamente morale. Ancora per gran parte dell'Ottocento, le sue cause furono ricercate nella povertà, nel vizio e nella corruzione derivanti dal progresso e dal lassismo dell'emergente borghesia. Le stigmate esibite nel fisico e nella mente dagli individui colpiti da forme degenerative erano un effetto della dissoluzione che, nonostante il progresso, aveva colpito la società. L'esito fatale di questo processo ricadeva sugli individui appartenenti ai due estremi sociali, i più miseri e i nobili. Se appartenevano agli strati più bassi erano inclini alla perversione e al decadimento perché dediti all'alcolismo, alle droghe e alla promiscuità; nell'aristocrazia, invece, la causa di tutti i mali era la consuetudine delle unioni consanguinee. A entrambe le categorie si rivolgevano i programmi d'igiene pubblica che, facendo leva sulle prospettive nefaste di degradazione e decadenza dell'umanità, miravano al risanamento fisico, morale e intellettuale. Morel fu il campione di questo atteggiamento della classe medica. Egli intendeva cogliere «le principe général qui préside à l'évolution rigoureuse des faits pathologiques ${ }^{38}$. Così finì per raggruppare, senza troppe fini distinzioni, l'insieme multiforme di pazienti ipocondriaci, imbecilli, idioti, isterici ed epilettici sotto la comune etichetta di un'alienazione rivelatrice dei segni dell'enchaînement fatal tipico della dégradation progressive. In realtà, molti di quei fenomeni morbosi con l'ereditarietà avevano poco da spartire; ma l'idea che fossero "ereditarie" la tisi e la sifilide, la gotta e la scrofula, aveva preparato il terreno agli psichiatri. Se nell'uomo - essere vivente dotato di capacità intellettuali e morali peculiari rispetto agli altri organismi - il miglioramento della specie era ottenibile soprattutto grazie alle funzioni del cervello, dove - se non in questo organo - si annidavano i "germi" più insidiosi delle malattie ereditarie, colpevoli di ostacolare la progressione verso l'alto? E quali facoltà, se non quelle psichiche, potevano manifestare i segni più vistosi delle aberrazioni ereditarie?

\section{Gli alienisti e gli slittamenti di paradigma}

Forse ancor più che le discussioni (e gli esperimenti) di medici, naturalisti e ibridatori, al concetto di "eredità biologica" contribuirono le idee degli alienisti francesi. Il concetto di ereditarietà si andava trasformando in un passepartout per gli psichiatri, interessati all'evoluzione progressiva dei fenomeni patologici e di taluni caratteri negativi del corpo, ma soprattutto della psiche. Dall'indagine sulla trasmissione ereditaria traeva origine una teoria della degenerazione nella specie umana, che, a sua volta, si ramificava in un insieme di riflessioni etico-sociali sullo sviluppo delle razze e della società.

(37) Cfr. L. Cartron, Degeneration and "Alienism" in Early Nineteenth-Century France, in Heredity Produced cit., in particolare pp. 155-174.
(38) B.-A. Morel, Traité des maladies mentales, Paris, Masson, 1860, p. 516. 
Nell'opera degli alienisti si concretò un fondamentale cambiamento, quello che in termini kuhniani si definirebbe uno spostamento di paradigma. Nel decennio precedente la pubblicazione dell'Origin di Darwin, ad opera degli psichiatri francesi ebbe luogo il passaggio dall'idea di trasmissione ereditaria a quella di un vero e proprio meccanismo degenerativo. Il concetto di tipo primitivo, e soprattutto l'idea che la degenerazione fosse di fatto inseparabile da una deviazione dal tipo primitivo, costituirono i fondamenti del citato Traité des dégénérescences di Morel, all'epoca primario della casa di cura per alienati di St.-Yon (Seine-Inférieure). Morel, che aveva condiviso gli studi medici con Claude Bernard ed era diventato segretario alla Salpêtrière del citato psichiatra Falret (padre) ) $^{39}$ aveva già pubblicato alcuni studi clinici sull'alienazione e su svariati stati anormali. A suo parere, l'insieme dei disturbi mentali era in costante aumento in Europa a causa del dilagante male fisico e morale, come attestava il numero crescente di suicidi, delitti, precocità nel crimine e imbastardimento della razza. Egli si proponeva di studiare l'origine, le cause e la formazione delle varietà morbose nella specie umana, di darne una classificazione indicando le misure profilattiche e igieniche al fine di migliorare lo stato intellettuale, fisico e morale dell'umanità. Le sue fonti erano la storia naturale e la fisiologia del primo Ottocento, i testi di Cuvier e Buffon mediati da Flourens. Ma era debitore anche nei confronti degli scritti non frenologici di Franz-Joseph Gall, che gli era noto attraverso Henri de Blainville, e conosceva le tesi di Blumenbach e di Alexander von Humboldt. E se dalle dottrine di Pinel, Esquirol, Joseph Dacquin e Fodéré aveva ricavato l'idea che «l'étude de l'homme physique ne peut s'isoler de l'étude de l'homme moral» ${ }^{40}$, attraverso la Société Médico-Psychologique aveva anche recepito il succo delle concezioni di missionari, filosofi ed economisti. Fondata da Baillarger a Parigi nel 1852, questa società infatti aveva come obiettivo la fusione dei diversi saperi medico-psicologici nello studio del genere umano.

Il clima, l'alimentazione e l'addomesticamento potevano influire sulla trasformazione delle specie animali, provocando alterazioni e degradazione, senza però formare nuove specie. Morel riteneva invece che nell'uomo le modificazioni morbose ingenerassero un'autentica deviazione dal tipo normale, la dégénérescence, culminante nella "condition invariable" della trasmissibilità ereditaria, fatale per le generazioni avvenire $^{41}$. Alla fine, i più degenerati erano impotenti a riprodursi. E se pure tra le cause di degenerazione erano comprese l'intossicazione per alcolismo o veleni (minerali e vegetali, tra cui l'hashish, i cui effetti erano stati recentemente indagati da Jacques Moreau de Tours ${ }^{42}$ ), la pellagra, l'azione morbifica delle terre paludose, un peculiare interesse rivestivano le forme di alienazione mentale conseguenti a un'affezione morbosa o a un temperamento malato, espressione di una lesione funzionale del sistema nervoso ${ }^{43}$. Tuttavia, la degenerazione derivava non tanto da infermità congenite o acquisite alla nascita, quanto piuttosto da un'influenza ereditaria, e questo era il fattore più potente di trasmissione. "Ereditaria" era non soltanto la malattia trasmessa dai genitori alla prole, ma anche la simple transmission di disposizioni organiche, che

(39) La biografia di Morel si trova in D. PICK, Faces of Degeneration: a European Disorder, c.1848-c.1918, Cambridge, Cambridge University Press, 1993 , p. 44 ss.

(40) B.-A. Morel, Traité des dégénérescences de l'éspèce bumaine, Paris, Baillière, 1857, p. XIV.

(41) Ivi, p. 34

(42) Allievo di Esquirol, Moreau aveva sperimentato gli effetti di questo allucinogeno in un viaggio in Asia Minore durato tre anni, intrapreso nelle vesti di accompagnatore di un paziente del suo maestro. Anche Moreau si domandava se la civilizzazione favorisse l'alienazione mentale, ed era arrivato alla conclusione che in Oriente l'incidenza delle malattie mentali era più bassa che in Europa, benché il fanatismo religioso fosse causa di follia. Gli esiti di questo viaggio apparvero nelle Annales Médico-Psychologiques (I, 1843, pp. 104-132) e successivamente in Du Hachisch et de l'Aliénation Mentale, Paris, Fortin, Masson et Cie, 1845.

(43) Morel, Traité des dégénérescences cit., pp. 53 ss. 
a loro volta diventavano nella progenie il punto di partenza di trasformazioni patologiche, tra di loro enchaînées e reciprocamente dipendenti e foriere di nuove entità morbose di ordine fisico e morale ${ }^{44}$. A questa concatenazione non si sfuggiva, perché soggetta a leggi fisse e inconfutabili, culminanti nella legge universale della «double fécondation», dei mali fisici e morali insieme, la cui azione congiunta si esemplificava nell'alcolismo, patologia nella quale all'intossicazione del genitore si sovrapponeva un quadro di miseria morale e materiale.

Anche Morel rinviava agli studi degli agronomi e degli allevatori. Questi avevano da tempo intuito l'importanza degli incroci, ma anche che, persistendo l'accoppiamento tra consanguinei, «tout s'éteint»: specie, razza, salute, fecondità, vitalità ${ }^{45}$. Di fatto, però ogni paragone tra uomo e animali era impossibile. A differenza degli altri viventi, l'uomo non è strumento passivo di un agente modificatore, bensì oppone la propria libertà. Abbandonate a se stesse, le razze artificiali «disparaissent» e non tardano «à retourner au type primitif», in quanto non riescono a vivere allo stato di natura ${ }^{46}$. Non così per l'essere umano, nel quale la consanguineità causa l'imbastardimento della specie, ma soprattutto l'aumento progressivo di ogni sorta di infermità fisica e mentale fino all'impotenza e alla morte sempre più ravvicinata di tutti i suoi prodotti. Morel annoverava le testimonianze degli agricoltori e dei fisiologi, molti dei quali già citati da Lucas, tra cui John Sebrigt ${ }^{47}$, John Sinclair, Girou de Buzareingues, Houdeville, e numerosi casi di animali tenuti in cattività. A causa di azzardati accoppiamenti consanguinei, si era addirittura provocata l'estinzione di alcune pregiate razze equine, causando - secondo il parere dei veterinari francesi Louis-Furcy Grognier e Jean-Baptiste Huzard - la scomparsa di uno dei più antichi allevamenti inglesi ${ }^{48}$.

Nel genere umano l'imbastardimento progressivo e l'estinzione della razza erano effetto dei matrimoni consanguinei. L'ereditarietà era causa di almeno $1 / 5$ dei casi considerati di alienazione, come comprovavano anche le statistiche elaborate da studiosi stranieri ${ }^{49}$. Da questi raffronti emergeva che la trasmissione ereditaria dell'alienazione, predominante per via materna, poteva dar origine a fenomeni di intermittenza, saltando talvolta una generazione. Veniva ammessa la correlazione con epilessia, isteria e ipocondria; i fenomeni di enchaînement fatal culminanti nel suicidio si manifestavano anche per via collaterale, mentre non pareva dimostrabile la trasmissione «d'un genre à un autre» tra le differenti malattie ereditarie, per esempio tra la tisi e la follia. Con un debito nei confronti all'umoralismo, Morel riconosceva infine che anche il temperamento nervoso poteva considerarsi ereditario e predisponente a svariate patologie, dall'isteria alle convulsioni, dalla melancolia alla follia. La parabola discendente della degenerazione si poteva spezzare con il «traitement moral» praticato nei manicomi. Emanazione della legge divina e universale, la legge morale non

(44) Ivi, p. 565.

(45) Ivi, p. 527.

(46) Ivi, p. 507.

(47) Nel Nouveau Dictionnaire d'Histoire Naturelle appliquée aux Arts, à l'Agriculture, à l'Économie Rurale et Domestique, à la Médecine etc. par une Société de Naturalistes et d'Agriculteurs (Tome XXVIII, Paris, Deterville, 1819) Jacques E. de Sève citava le osservazioni di Sebrigt sulla degenerazione nei cani e nei volatili da cortile con individui «tra loro ravvicinati» per parentela. Nei maiali, le femmine diventavano presto sterili e quelle che partorivano, generavano una prole così debole che moriva quasi subito. Questo giudizio era condiviso dagli allevatori e dai coltivatori, i quali osservavano énervation, perdita di capacità riproduttive $\mathrm{e}$ deterioramento della razza.

(48) MOREL, Traité des dégénérescences cit., p. 527. Morel alludeva presumibilmente a Robert Bakewell, il famoso allevatore inglese citato anche da Darwin. Su ibridazione e selezione praticate per il miglioramento delle specie, si veda la storia dei Vilmorin, celebre famiglia di coltivatori francesi in J. Gayon e D.T. Zallen, The Role of Vilmorin Company in the Promotion and Diffusion of the Experimental Science of Heredity in France, 1840-1920, «Journal of the History of Biology» XXXI, 1998, pp. 241-262.

(49) G. Mann Burrows (Commentaries on the Causes, Forms, Symptoms, and Treatment, Moral and 
si applicava in egual misura presso tutte le popolazioni, a causa del diverso grado di civilizzazione raggiunto, e persino nelle nazioni più progredite si assisteva al pauroso decadimento di talune classi sociali. La moralizzazione delle masse era un lavoro gravoso, il recupero «lent et difficile»; ma nel suo trattato di igiene fisica e morale, Morel non disperava di ottenere la régénération.

Con il Traité des maladies mentales del 1860 le idee sulla degenerazione ebbero una notevole diffusione in ambito psichiatrico ${ }^{50}$. A un modello diagnostico analogo si richiamava anche Griesinger. Irrequieto studioso delle malattie mentali ${ }^{51}$, alle quali si era accostato partendo da un'impostazione clinica oltre che anatomo-patologica, Griesinger fu uno psichiatra sui generis: la sua opera si attuò in una fase di trasformazione della clinica psichiatrica che, dalla medicina romantica, approdava al materialismo posivistico ${ }^{52}$. Altri hanno definito il suo approccio "razionale" o, all'opposto, "empirista", e c'è chi ha visto nella difficoltà di liberarsi dall'eredità filosofica hegeliana un tratto tipico della generazione degli psichiatri tedeschi di fine Ottocento. Griesinger rinviava alla letteratura e alle statistiche citate da Morel, condividendone l'impostazione. Pur ammettendo il ruolo di una «causa esterna insignificante», dalla quale "improvvisamente" può sprigionarsi la malattia mentale, riconosceva l'importanza della costituzione 《cosiddetta nervosa» ${ }^{53}$, identificabile nel cervello o nel midollo spinale. Essa manifesta segni inequivocabili nei correlati fisici, dall'iperestesia alla perdita di fluidi corporei, dall'irritazione dei genitali alle emorroidi. Ed è ben visibile nella sfera mentale, caratterizzata da eccessiva sensibilità, instabilità, sviluppo intellettuale discontinuo e "sproporzionato", riscontrabile anche negli artisti e nei geni. Non di rado, alla "circostanza predisponente" della costituzione nervosa si associa una causa "reale", che consiste o in una malattia fisica o in un'influenza morale negativa, la quale a sua volta innesca il passaggio dalla semplice disposizione al disordine in atto.

\section{Lamarck, sistema nervoso ed ereditarietà}

Con l'introduzione del significato "tecnico" del termine dégénéré da parte di Morel si sanciva una profonda frattura con il passato. A Buffon spettava pur sempre il merito di aver introdotto il concetto, ma per i naturalisti francesi il meccanismo della degenerazione restava soltanto uno dei vari marchingegni a sostegno della legge dell'unità del genere umano. Benché deviati dal tipo primordiale (bianco), negri e lapponi propagavano i caratteri della grande famiglia umana. Morel invece si attribuiva la prerogativa di aver connotato in senso negativo la degenerazione: si trattava di una vera e propria deviazione maladive che avrebbe condotto all'estinzione degli individui di quel ramo ${ }^{54}$.

Medical, of Insanity, London, Underwood, 1828) ammetteva l'ereditarietà per i 6/7 dei casi; Esquirol ne contava 40 su 265; per Joseph Guislain il peso era di $1 / 4$, e di $1 / 5$ secondo Maximilian Jacobi, il teorico della psichiatria somatica e direttore della casa di cura di Siegburg (Beobachtungen über die Pathologie und Therapie der mit Irresein verbundenen Krankheiten, Elberfeld, Schönion, 1830).

(50) Paris, Masson, 1860.

(51) Dopo alcune esperienze in manicomi tedeschi e francesi, Griesinger fu per circa tre anni presidente della commissione medica al Cairo del Kédivé Abbas, e poi fece ritorno in
Germania, Svizzera e infine a Berlino, dove diresse il dipartimento delle malattie nervose della Charité (1865).

(52) Cfr. A.L. Mishara, Commentary on "Wilhelm Griesinger", «Philosophy, Psychiatry, \& e Psychology» III, 1996, pp. 165-167; E. ACKERKNECHT, Kurze Geschichte der Psychiatrie, Stuttgart, Enke, 1959, tr. ingl. col titolo A Short History of Psychiatry, New York - London, Hafner, 1959, p. 63.

(53) Griesinger, Mental Pathology cit., p. 111.

(54) Morel, Traité des dégénérescences cit., pp. $14-15$. 
Ma che lo ammettesse o no, anche Morel era in debito con i naturalisti: egli aveva seguito l'insegnamento di Henri de Blainville ${ }^{55}$, a sua volta discepolo di Cuvier e suo successore al "Muséum national d'Histoire naturelle". Da Blainville aveva ereditato l'avversione per il trasformismo lamarckiano ${ }^{56}$. E questo spiegherebbe perché il nome di Lamarck compaia raramente tra le fonti degli alienisti a proposito dell'ereditarietà. Eppure in diversi luoghi della Philosopbie zoologique Lamarck aveva trattato il binomio «altération organique ou fonctionnelle» e «altération des facultés morales et intellectuelles», prendendo l'avvio da quei passi in cui Cabanis stabiliva una corrispondenza tra la presenza di condizioni psichiche quali la tristezza e la melanconia «sans sujet réel» e l'alterazione nelle viscere dell'addome in pazienti che lamentavano stati d'animo dolorosi. Queste alterazioni potevano far insorgere un penchant alla malinconia, anche senza motivo, e «à la vérité, la génération peut transmettre une disposition des organes, en un mot, un état des viscères propre à donner lieu à tel tempérament, telle disposition, enfin, tel caractère» ${ }^{57}$.

Però Cabanis si era «étendu trop loin» nel ritenere che le viscere potessero concorrere alla formazione del pensiero. Pur convinto della "verità costante" delle due leggi fondamentali dell'esercizio e della trasmissione alle generazioni future dei cambiamenti acquisiti, Lamarck era molto cauto circa gli effetti che questi meccanismi potevano avere su organi particolari come quelli che formano gli emisferi cerebrali e danno origine alle facoltà intellettuali. Infatti riteneva che Franz Joseph Gall, il quale si era proposto di applicare quei principi all'organo dell'intelligenza, ne avesse abusato, traendone conseguenze illegittime, in quanto soltanto in casi eccezionali le facoltà superiori possono esibire i segni esterni indubitabili della trasmissione dei caratteri modificabili o acquisiti. E se pure alla nascita si ereditano speciali tendenze, nel caso in cui non si esercitino abitualmente e fortemente le facoltà pertinenti, non conseguirà alcuno sviluppo dell'organo particolare che ne esegue gli atti.

Lamarck era uno strenuo sostenitore del ruolo delle circostanze atte a favorire l'espressione delle disposizioni. Soltanto negli animali inferiori «la génération transmet, presque sans variation, l'organisation, les penchans, les habitudes, enfin, tout ce qui est le propre à chaque race ${ }^{58}$. Nell'uomo, invece, è soprattutto l'educazione che determina la direzione e il movimento del fluido nervoso. Sogni, delirio, follia possono seguire a un imperfetto controllo del fluido nervoso da parte del sentiment intérieur nell'ipocefalo, per esempio a causa di un trauma o di una forte emozione o in conseguenza di atti disordinati di memoria. Il fatto che alcuni individui commettano atti di pazzia soltanto relativamente a certi oggetti (in genere sempre gli stessi) fa pensare che in essi vi sia un particolare dérangement non già di tutto l'ipocefalo, bensì soltanto di certe sue parti, mentre le altre conservano la propria integrità ${ }^{5}$. Questi fenomeni sarebbero effetto di atti fisici dipendenti dall'organizzazione, dalle circostanze e dalle cause esterne (sempre di natura fisica) ${ }^{60}$.

Alla base di questa concezione del rapporto tra "innato" e "acquisito" era una teoria fisiologica che vedeva nel sistema nervoso un intrico di fibre, canali e cavità interne percorsi da un fluido sottile, invisibile, dal movimento rapido, ma soprattutto «très-analogue au fluide éléctrique». Se agitato, questo liquido produceva sensazio-

(55) S. Legrand, Portraits du dégénéré en fou, en primitif, en enfant et finalement en artiste, «Methodos» (en ligne) III, 2003.

(56) Il rapporto tra Blainville e Lamarck sembra molto più sfaccettato di quanto sostiene Legrand. Cfr. T.A. AppeL, The Cuvier-Geoffroy Debate: French Biology in the Decades before Darwin, Oxford, Oxford University Press, 1987, p. 38 e passim.
(57) J.-B.-P.-A. de Monet de Lamarck, Philosophie zoologique, Paris, Dentu, 1809, Troisième partie, cap. IV, p. 293.

(58) Ibid.

(59) Ivi, p. 430.

(60) Ivi, p. 434. 
ni, sentimenti ed emozioni. Di impronta meccanicistica era l'idea che ai movimenti irregolari del fluido nervoso nel cervello fossero imputabili le idee sconnesse e disordinate, i pensieri strani ed eterogenei. L'insieme dei fenomeni nervosi, normali e patologici, espressivi di sensibilità e intelletto, si spiegava attraverso una "dynamique organique", conseguente a movimenti muscolari e nervosi, e quindi all'uso e disuso delle parti ${ }^{61}$. Lamarck derivava queste idee dalla fisica e dalla chimica, discipline che forse ancor più della paleontologia costituivano, insieme con la botanica, il suo ambito professionale. All'epoca, il galvanismo aveva ormai preso piede: «Les expériences galvaniques nous ont appris que le fluide électrique qui agit si facilement sur les nerfs, en se transmettant avec célérité dans leur masse, a nécessairement beaucoup d'analogie avec le fluide nerveux», e numerosi sono i luoghi in cui Lamarck cita Volta e i suoi esperimenti ${ }^{62}$. I fenomeni elettrici si inquadravano nella dottrina halleriana dell'irritabilità, che spiegava «des mouvemens de certaines des parties [du] corps», fenomeno che pareva «résulter d'un galvanisme particulier et local, dû à la composition chimique des parties qui peuvent l'offrir, et qui produit certains mouvemens dans ces parties, à chaque provocation d'une cause excitante» ${ }^{63}$. L'azione muscolare era una risultante dell'influsso nervoso, «une émission de fluide nerveux», che per esercitare la propria azione necessita dell'irritabilità, senza la quale, l'eccitazione nervosa sarebbe «de nul effet» ${ }^{64}$.

In questo quadro di fisiologia materialistica che riduceva i fenomeni mentali a fatti fisici e meccanici, la concezione lamarckiana del fluido nervoso non sembrerebbe avere particolari legami né con la teoria dell'ereditarietà dei caratteri acquisiti né con l'ipotesi della degenerazione, che di lì a breve sarebbe stata formulata dagli alienisti. Eppure, se si esamina l'opera degli psichiatri francesi di terza generazione, si scopre che, apparentemente indipendenti, i percorsi concettuali della medicina della mente erano quelli praticati da Lamarck.

\section{La degenerazione dopo Morel}

Fin dalla dottrina umoralistica dei temperamenti la costituzione nervosa era considerata ereditaria e predisponente a una serie di malattie mentali. Ma nell'Ottocento, seppur lentamente, le cose stavano cambiando. Già fin nella seduta del 29 giugno 1857 della Société Médico-Psychologique, si erano levate osservazioni critiche circa il binomio "eredità-degenerazione", la cui portata cominciava a esser ridimensionata. Se «le mot dégénérescence est un mot anthropologique», allora il fenomeno ereditario equivaleva a «une complète déviation du type primitif». Forse Baillarger aveva esagerato nell'insistere sull'influenza egemone dell'ereditarietà («qui dit dégénérescence dit hérédité»). Che una madre fosse isterica non giustificava la scrofula nel figlio: «[...] on n'y comprend pas la transmission d'un genre à un autre» e, di conse-

(61) P. Consi, Oltre il mito. Lamarck e le scienze naturali del suo tempo, Bologna, il Mulino, 1983, pp. 214-216.

(62) J.-B.-P.-A. De Monet de Lamarck, Recherches sur l'organisation des corps vivans et particulièrement sur son origine, sur la cause de ses développemens et des progrès de sa composition, et sur celle qui, tendant continuellement à la détruire dans chaque individu, amène nécessairement sa mort; précédé du discours d'ouverture du cours de zoologie, donné dans le Muséum national d'Histoire Naturelle, Paris, Maillard, 1802, pp. 164, 176, 194, e Philosophie zo- ologique cit., pp. 17, 240, 242-44. Una voce fuori dal coro era quella di Erasmus Darwin, il quale riteneva che tra lo spirito animale, l'aetherial fluid, e l'elettricità animale di ispirazione galvanica vi fosse soltanto analogia, cfr. Zoonomia, London, Johnson, I, 1801, pp. 10 e 37; su questo punto cfr. Brain, Mind and Medicine. Neuroscience in the $18^{\text {th }}$ Century, a cura di H. WhitTaker, C.U.M. Smith and S. FINGER, New York, Springer, 2007, pp. 23-24.

(63) LamarcK, «Irritabilité», in Nouveau Dictionnaire d'Histoire Naturelle, vol. XVI, 1817, p. 396.

(64) Ivi, p. 401. 
guenza, da una perversione organica non si potevano generare indifferentemente tutti i tipi di malattia. La seduta si era conclusa con l'ammissione che la degenerescenza era questione «très complèxe», al punto da costituire una sorta di enciclopedia, in cui era impossibile qualsiasi ordinamento ${ }^{65}$.

Quarant'anni più tardi regnava ancor più confusione: come si è visto, al quinto convegno degli alienisti francesi c'era chi lamentava l'abuso dei concetti di predisposizione ereditaria e di degenerazione. Ma non mancava chi era tuttora entusiasta della teoria delle dégénérescences: après Morel tutto era cambiato. Soppiantate le vecchie tesi umoralistiche sulla follia, a Morel andava il merito di aver fatto un passo avanti anche rispetto alle monomanie propugnate da Esquirol. Con intenti puramente classificatori e facendo appello ai sintomi, Esquirol era responsabile di un'indebita proliferazione di entità morbose, arrivando a confondere «les choses les plus différentes» e a separare «psychoses de même essence» ${ }^{66}$. La stagione delle monomanie - intellettuali, affettive, istintive, sensoriali ecc. - ora si avviava al declino. Fin dal 1857 Morel invece aveva cercato di raggruppare le malattie mentali per mezzo di un metodo eziologicoclinico in luogo di quello puramente sistematico. Questo passaggio fondamentale nella nosografia mentale si concretava grazie a un nuovo accorgimento metodologico: l'osservazione del fenomeno palpable era completata con l'indagine del substrato mentale, che ora veniva «passé au crible» ${ }^{67}$.

Valentin Magnan e Paul-Maurice Legrain, autori di Les Dégénérés, avevano maturato una certa esperienza in fatto di ereditarietà e alcolismo. Prima di LucasMoreau de Tours-Morel, non si era capito che, nel ripetersi della follia nella progenie, si configurava una nuova forma di stato mentale caratterizzata da una tara ereditaria. Un tempo, i fatti di eredità erano semplicemente un esempio di trasmissione (più o meno diretta) di abitudini e condizioni simili secondo età, sesso ecc. Morel invece per primo aveva formulato l'idea scientifica di una trasformazione di specie, cioè un fenomeno patologico, a seguito del quale «les descendents ne présentent plus au but d'un certain nombre de générations les mêmes attributs que les ascendents, mais de nouveaux attributs physiques et intellectuels fixes, immuables, qui les différencient du type commun de l'espèce et qui en font de nouveaux êtres» ${ }^{68}$ : les dégénérés.

Trent'anni dopo l'introduzione di queste idee, non mancavano le dissidences. Consapevoli che «les classifications actuelles [sont] presque aussi nombreuses que les aliénistes ${ }^{69}$, Magnan e Legrain intendevano superare la rigida suddivisione di Esquirol tra stati primari e secondari, tra disturbi affettivi e intellettuali. Nelle malattie ereditarie rientravano tutte le forme di follia, comprese quella "ragionante", lucida, e quella morale. Persino lo psicopatologo Richard von Krafft-Ebing e lo psichiatra vittoriano Henry Maudsley erano convinti che queste forme di follia avessero un'origine degenerativa. E se ancora nel 1885, un lavoro dello stesso Magnan presentato alla Société Médico-Psychologique sulle aberrazioni e perversioni sessuali aveva innescato una lunga discussione sulla follia ereditaria ${ }^{70}$, ora tutte le polemiche classificatorie potevano essere superate distinguendo tra "héréditaire" e "dégénéré". In effetti, sul piano clinico neppure Morel sembrava aver fatto progressi significativi, poiché alla degenerazione riconduceva tutte le forme di alienazione, tranne il delirio di persecuzione, derivante da uno stato precedente di nevrosi ipocondriaca. Occorreva invece

(65) Sociétés savantes. Société médico-psychologique, Séance du 29 juin 1857, «Annales médicopsychologiques» III, 1857, pp. 626-627.

(66) V. Magnan e P.M. Legrain, Les Dégénérés: état mental et syndromes épisodiques, Paris, Rueff, 1895, p. 10.
(67) Ivi, p. 11.

(68) Magnan e Legrain, Les Dégénérés cit., p. 12.

(69) Ivi, p. 19.

(70) V. Magnan, Des anomalies, des aberrations et des perversions sexuelles, Paris, Delahaye et Lecrosnier, 1885. 
insistere sull'aggravamento progressivo della degenerazione che mette capo all'annientamento della specie. La sterilità era il sigillo definitivo, che della degenerazione sanciva la natura di patologia non regressiva o di semplice anomalia con carattere di reversione.

Verso la fine del secolo, dunque, il legame tra eredità e degenerazione si era spezzato. L'antropologia aveva mostrato che tutte le specie, compresa quella umana, tendono alla perfezione. Per mezzo di nutrizione e riproduzione, anche l'uomo "marcia" dal tipo meno perfetto al più perfetto, arrivando a lottare per questo fine. Come si spiegava allora che, a un certo punto, avesse origine la degenerazione? Senza mai citare esplicitamente né Lamarck né Darwin, gli psichiatri francesi di fine Ottocento avevano assimilato la lezione dell'evoluzionismo, riconoscendo che la perfettibilità è «une qualité de tout être qui évolue normalement» ${ }^{71}$. La degenerazione si presenta allora come un movimento di progressione al contrario, da uno stato più perfetto a uno meno perfetto, a seguito dell'intervento di una causa suscettibile di contrastare il doppio movimento naturale verso la conservazione della specie e dell'individuo. Esistono cause degeneratrici, le cui influenze nocive si esercitano a prescindere dai tipi ancestrali generatori e danno origine a un «nouveau type [qui] naît différent» dai suoi progenitori, portatore di attributi denotanti uno stato progressivo di inferiorità psicofisica $^{72}$.

Il nuovo «sistema» proposto da Magnan e Legrain consisteva in una echelle double, la cui porzione ascendente raffigurava l'evoluzione regolare della specie, mentre quella discendente l'evoluzione «dans le sense de la dégénérescence» ${ }^{73}$. Le origini della prima sono indeterminate e si perdono nella notte dei tempi, a un certo punto $A$ corrisponde la comparsa della specie umana, i cui tipi normali recano in sé, in poten$\mathrm{za}$, i germi del miglioramento progressivo, verso un punto indefinibile dello stato di maggior perfezione. Ciò non impedisce tuttavia che, nel corso di questa evoluzione, si possano trovare in svariati punti, dilazionati, degli ostacoli, veri e proprifacteurs de déséquilibration. Si tratta di agenti distruttori dell'equilibrio biologico, che operano incuranti della struttura psicologica e morale che la specie ha raggiunto al momento della loro comparsa. La degenerazione può «éclater» in un punto qualsiasi della storia dell'uomo e, da questo momento, il tipo degenerato avvierà la propria marcia retrograda verso un punto $Z$, nel quale si estingue. $Z$ si presenta perfettamente simmetrico ad $A$, e in esso si colloca l'idiota, «être instinctif par excellence, [...] machine automatique» ${ }^{74}$, che riassume in sé tutte la serie di degradazioni successive subite dai suoi ascendenti nei vari punti in cui si situano i fattori della degenerazione, così come in $A$ si trova il tipo che comprende in sé tutti i caratteri dell'evoluzione normale e regolare della sua discendenza. E se nell'elenco delle cause degeneratrici, suddivise tra fisiche (telluriche e climatiche) e morali la classificazione non si discostava da quelle precedenti, ora non mancava una presa di posizione contro Morel. La sua era stata una mera classificazione metodica, strumento sociologico improduttivo di rimedi. Eliminando le classificazioni, la clinica aveva chiarito che il tipo del degenerato non varia, quale che sia la causa del suo stato. I degenerati non sono esseri semplicemente regressivi, giacché in tal caso non devierebbero dal tipo normale. Pur costituendo un arretramento verso uno stato meno perfetto, regressione e reversione sono processi che comunque finiscono per ritornare agli stati normali. Questi fenomeni impliche-

(71) Magnan e Legrain, Les Dégénérés cit., p. 75 .

(72) Ivi, p. 76
(73) Cfr. H. Wallon, L'idée de dégénérescence mentale, «L'année psychologique» XX, 1930, pp. 190-196, p. 195.

(74) Magnan e Legrain, Les Dégénérés cit., p. 165. 
rebbero tutt'al più un ritardo nel corso dell'evoluzione, ma mai la creazione di un nuovo tipo morboso, che non possiede più i mezzi per rigenerarsi.

Morel era un inguaribile ottimista ${ }^{75}$, illuso circa la possibilità di una rigenerazione, grazie all'apporto di sangue nuovo da matrimoni non consanguinei. Al contrario, l'esperienza insegnava che i degenerati sono soliti unirsi tra loro in matrimonio, ragion per cui i medici e la società hanno il dovere di contrastare la propagazione della degenerazione, autentica piaga sociale ${ }^{76}$. Benvenuto allora l'intervento del medicosociologo, che con una politica di rigorosa igiene sociale (prophilaxie sociale) estirpa il male alle radici, limitandone le cause di natura biologico-evolutiva e sociale, più insidiose quando agiscono in gioventù su un organismo in via di sviluppo. In età adulta, ai fattori collettivi, come guerre e carestie, si aggiungono le malattie professionali e quelle conseguenti agli eccessi di una civilizzazione avanzata, l'alcolismo e la dipendenza da stupefacenti.

La biologia consentiva di delineare il percorso dell'evoluzione, nella quale l'uomo "idealmente normale" è «suffisamment armé pour la lutte», in grado di preservare l'equilibrio biologico per sé e per la progenie ${ }^{77}$. La specie umana ha sviluppato organi sempre più adatti alle funzioni finalizzate al successo riproduttivo, e l'organismo mette capo meravigliosamente al doppio fine della riproduzione e della conservazione della specie ${ }^{78}$. Ma allorquando, in conseguenza di un disordine organico, viene compromessa una funzione importante e si rischia di perdere l'equilibrio biologico, l'essere degenerato scompare o non è più in grado di riprodursi. Sovente si tratta di cattive disposizioni organiche congenite: le influenze ereditarie formano un insieme complesso, un accumulo di tare di ordine diverso, acquisite dagli ascendenti in una serie indefinita di generazioni, ma combinate sotto forma di stigmate psicofisiche. Pur trattandosi di una trasmissione morbosa ereditaria, la degenerazione ha quasi sempre un'origine prima, identificabile con un'influenza accidentale. Questa o si estingue nel soggetto o diventa costituzionale, autentico germe di infezione che si riproduce nella discendenza. I degenerati sono individui dal sistema nervoso fragile, di scarsa resistenza, instabili, portatori di tare che discendono dai progenitori, ma che si accrescono e aggravano nel corso della vita.

Per contrastare questa figura perniciosa per la specie e destinata a une guérison impossible ${ }^{79}$, si impongono mezzi eccezionali sul piano dell'educazione: fisica, intellettuale, morale e professionale. Nel lavoro consiste l'azione moralizzatrice per eccellenza e, di conseguenza, su tutti i tipi di educazione predominerà l'insegnamento professionale, finalizzato a insegnare mestieri rurali e manuali, che allontanino il degenerato dai centri urbani pullulanti di cause dissipatrici. Paradossalmente, se il progresso genera le cause della decadenza, la società dovrà a sua volta premunirsi, moltiplicando i rimedi e confidando nel ruolo di una nuova figura professionale: il medico-pedagogista-sociologo.

\section{Epilogo}

Nel 1876 il naturalista Giuseppe Canestrini tradusse in italiano la seconda edizione, uscita l'anno precedente, della Variation darwiniana. In quest'opera, dopo aver riconosciuto che il tema dell'ereditarietà era meraviglioso e immenso ${ }^{80}$, Darwin

(75) Ivi, p. 233.

(76) Ivi, pp. 218 e 233-235.

(77) Ivi, p. 88.
(78) Ivi, pp. 87-88 e 91.

(79) Ivi, p. 218.

(80) C. DarwIN, The Variation of Animals and 
elencava gli autori che se ne erano occupati. Oltre a Lucas, che lo aveva affrontato in un'opera di 1562 pagine $^{81}$, anche altri medici e naturalisti vi avevano contribuito: primo fra tutti William Sedgwick in una serie di articoli apparsi tra il 1861 e il 1863 nella «British and Foreign Medico-Chirurgical Review». A questo punto, Darwin osservava che era «sorprendente» l'unanimità delle credenze dei veterinari di tutti i paesi a favore della trasmissione delle tendenze morbose, ma anche delle caratteristiche inerenti alla buona salute, al vigore e alla longevità. Purtuttavia concludeva che «il potere della trasmissione è estremamente variabile», giacché alcuni soggetti lo manifestano «in modo perfetto», mentre in altri è carente. E non sembrano esservi ragioni per queste differenze. L'unica certezza è che non tutti i caratteri sono trasmissibili, per esempio non lo sono gli effetti di mutilazioni o di lesioni. Inoltre, vi sono ostacoli all'ereditarietà, come per esempio talune circostanze particolarmente ostili, la variabilità conseguente alle mutate condizioni di vita, l'incrocio delle varietà nelle precedenti generazioni, che induce la tendenza della progenie a rassomigliare agli antenati piuttosto che ai genitori. Infine, più che alla degenerazione, Darwin pareva interessato all'effetto della regressione e al suo rapporto con le variazioni e gli incroci.

Sia pure con mille riserve, egli presentava l'ipotesi della pangenesi, secondo la quale talune "gemmule" o particelle mobili nel corpo sarebbero responsabili della trasmissione delle abilità acquisite. Frattanto, con esperimenti sulla trasfusione del sangue tra razze distinte di conigli, suo cugino Francis Galton formulava un giudizio fortemente negativo su questa dottrina. Nel prosieguo di questi esperimenti, Galton si aspettava di trovare le gemmule nel sangue, per quanto questa ipotesi non fosse del tutto suffragata, dal momento che era stata avanzata anche nel caso delle piante e di invertebrati senza circolazione sanguigna ${ }^{82}$. Inoltre, il fisiologo franco-americano Charles-Édouard Brown-Séquard fin dalla metà del secolo aveva dato inizio a serie sperimentali, nelle quali provocava attacchi epilettici alle cavie per mezzo di sezioni del sistema nervoso, in particolare sul midollo spinale e sul nervo sciatico e, a partire dal 1860, aveva osservato che la condizione epilettica e i cambiamenti conseguenti venivano frequentemente trasmessi alla prole delle cavie, alle quali erano state impartite quelle lesioni ${ }^{83}$. Darwin concludeva però che Galton, il quale era a conoscenza di questi risultati sperimentali, non fosse affatto convinto che gli effetti di lesioni o delle abitudini dei genitori potessero essere trasmessi alla discendenza, né che si avessero conseguenze sulle facoltà o sulla morfologia della progenie. Al problema dell'ereditarietà Galton aveva dedicato le ricerche di una vita, sotto il segno dell'idea che «le abilità naturali dell'uomo sono derivate per ereditarietà», secondo quanto si legge nell'incipit del capitolo introduttivo della seconda edizione di Hereditary Genius (1892) o come attestano le sue indagini sui gemelli, che costituivano un banco di prova per saggiare l'influenza combinata tra eredità e ambiente. Galton era al corrente, tra l'altro, degli studi di Moreau de Tours e di Théodule Ribot ${ }^{84}$, e aveva diffuso questionari e sondaggi per controllare gli effetti della dicotomia nature/nurture sulla progenie, ravvisabili nei soggetti che, benché allevati allo stesso modo, restavano dis-

Plants under Domestication, London, Murray, $1875^{2}$, I, cap. XII, p. 446.

(81) Ivi, p. 445.

(82) C. DARIWIN, Variation cit., tr. it. col titolo $\mathrm{La}$ variazione degli animali e delle piante allo stato domestico, a cura di G. Canestrini, Torino, Utet, 1876, cap. XXVII, n. 1, p. 688.

(83) Cfr. DARWIN, Variation cit., p. 468.

(84) D. Burbridge, Francis Galton on Twins, Heredity and Social Class, «British Journal for the
History of Science» XXXIV, 2001, pp. 323-340. L'opera di Moreau de Tours cui si allude è La psychologie morbide dans ses rapports avec la philosophie de l'histoire, ou de l'influence sur le dynamisme intellectuel (Paris, Masson, 1859). Del saggio di T. Riвот, L'Hérédité: Étude psychologique sur ses phénomènes, ses lois, ses causes, ses conséquences (Paris, Ladrange, 1873), Galton stesso aveva curato una recensione apparsa in «Academy» nel 1875. 
simili, o in coloro i quali, nati simili, a seguito di separazioni e differenze nell'educazione, manifestavano una graduale estinzione delle rassomiglianze.

Moreau de Tours aveva individuato somiglianze tra genio e follia. La sua opera fu una delle fonti dell'antropologia di Cesare Lombroso, che intendeva dimostrare che il comportamento criminale è ereditario. Per Moreau, addirittura anche il genio era una nevrosi, uno stato semimorboso del cervello ${ }^{85}$, nella genesi del quale aveva un ruolo determinante la predisposizione, cioè la costituzione organica dell'individuo. Ispirandosi tanto a Morel quanto a Moreau, lo stesso Lombroso concepiva il genio come una forma di degenerescenza, nella quale ravvisava un'espressione di regressione. $\mathrm{Ma}$, così facendo, confondeva i due concetti, perché mentre la prima consiste in un'alterazione anatomica di un organo che mette capo alla dissoluzione di una razza, la seconda è invece un arresto dello sviluppo, quindi lo stato di un processo evolutivo che però non riguarda la condizione di malattia di un organo o di un tessuto ${ }^{86}$.

La storiografia che di recente ha approfondito la diffusione del modello della degenerazione nella cultura (non solo europea) tra Otto e Novecento non ha mancato di sottolineare, da un lato, le simpatie lamarckiane di Darwin, e da un altro lato il debito di Lombroso nei confronti, non tanto di Darwin, quanto soprattutto degli alienisti francesi, di Galton e di Ernst Haeckel ${ }^{87}$. A questo punto, complice la circolazione delle idee di Lombroso negli Stati Uniti, il tema della degenerazione era destinato a ricongiungersi con la teoria dell'atavismo: ma mentre per gran parte degli alienisti la degenerescenza era una deviazione morbosa da un tipo originale destinata a culminare con l'estinzione, l'atavismo implicava un ritorno, una regressione a caratteri primitivi ancestrali. E su questi fondamenti poggiava l'interrogativo se la deviazione da una più perfetta linea evolutiva implicata dalla degenerazione rappresentasse la trasmissione di caratteri arcaici o la «devoluzione da uno stato evoluto a uno atavico», che poteva consistere anche nell'arresto dello sviluppo o nell'incapacità di completare il ciclo evolutivo ${ }^{88}$.

Nello stesso tempo, benché gli scienziati avessero esortato a non confondere i termini "eredità/degenerazione", nella cultura europea si andavano sviluppando, sia pure disordinatamente, le idee disseminate da quel binomio. Formulato in ambito medico-biologico, il paradigma interpretativo fondato su quella coppia di concetti si prestava a diventare una chiave di lettura del cambiamento nei processi psichici, nei fenomeni politico-sociali, nell'etica ecc. Per una delucidazione di quel rapporto sul piano biologico si sarebbe dovuta invece aspettare la teoria mendeliana e, successivamente, la rivoluzione della genetica. Ma questa è un'altra storia, che persino con l'impostazione di Galton e con l'idea della "continuità del plasma germinale" elaborata da August Weismann ha ben poco che vedere, perché all'epoca non poteva ancora essere noto che soltanto il gene rappresenta l'unità dell'ereditabilità.

GERMANA PARETI

Università degli Studi di Torino

(85) Moreau, Psychologie morbide cit., p. 465.

(86) Cfr. É. RaBAud, La génie et les théories de $M$. Lombroso, Paris, Mercure de France, 1908, p. 39.

(87) Cfr. Pick, Faces of Degeneration cit.
(88) D. SeItler, Atavistic Tendencies: the Culture of Science in American Modernity, Minneapolis, University of Minnesota Press, 2008, p. 61. 